\title{
Devastating Insomnia Experience Following General Anaesthesia for Mastoidectomy Surgery
}

\author{
LH Misron $^{\mathrm{a}}$, SNF Misron ${ }^{\mathrm{b}}, \mathrm{K}$ Misron $^{\mathrm{c}}$ \\ ${ }^{a}$ Department of Anesthesiology, Hospital Bintulu, Jalan Bkt Nyabau, Sarawak \\ ${ }^{b}$ Department of Psychiatry, Hospital Tawau, Sabah. \\ 'Department of Otorhinolaryngology - Head and Neck Surgery, Hospital Tawau, Sabah
}

ABSTRACT

Sleep disorder including insomnia is one of the complications of general anaesthesia. It is not uncommon and it is temporary but majority remains unnoticed and untreated. The effect of insomnia ranges from mild to severe, influencing both physical and mental health. Surgeon and anaesthetist need to identify this complication so that an appropriate treatment can be delivered. In complicated case, psychiatrist involvement is crucial. The treatment is symptomatic and temporary. We reported a case of distressful sleep disorder as a sequelae of general anaesthesia for mastoidectomy surgery. Subsequently after symptomatic treatment, he recovered completely and regained his normal sleep pattern.

Keywords: Anaesthesia; insomnia; paradoxical effect.

\section{INTRODUCTION}

General anaesthesia is performed daily all around the world. Many surgeries rely on general anaesthesia to be performed successfully. Apart from the surgical complications itself, general anaesthesia warranted attention for its minor and major complications of which one of them is sleep disorder. Sleep disorder affected many patients post surgery for many reasons such as paradoxical effect of anaesthetic agents, adjustment to hospital surrounding, pain, and psychological cause. The prevalence was as high as $55 \%$ in patients post abdominal, vascular and orthopaedic surgery done under general anaesthesia. ${ }^{1}$ In spite of its high prevalence, it is not uncommon for sleep disturbance to be overlooked despite of its potential devastating effect to the patients. Our

Corresponding author

Dr. Siti Nor Fadhlina Misron

Department of Psychiatry, Hospital Tawau,

Peti Surat 67, 91007 Tawau, Sabah

Email:drfadhlina@gmail.com patient presented with sleep disorder specifically severe insomnia following a right mastoidectomy surgery.

\section{CASE REPORT}

A 23-year-old man with no known medical illness underwent a right modified radical mastoidectomy under general anaesthesia for right cholesteatoma. The anaesthesia was performed without muscle relaxant as intraoperative facial nerve monitoring was used. Anaesthetic agents given on the day of operation were Intravenous Fentanyl $100 \mathrm{mcg}$, Intravenous Propofol 120mg, Intravenous Rocuronium $50 \mathrm{mg}$ for intubation, Intravenous Morphine $4 \mathrm{mg}$, and Intravenous Dexamethasone $8 \mathrm{mg}$. The operation was uneventful pre-, intra-, and post-operatively.

However, the next day he started to experience distressing hypnagogic hallucination every time he started to doze off at any time of the day. He saw people and animals moving around him and made noises. Although brief, it awakened him and he was unable to proceed with his sleep and tiredness sets in as he began to be deprived of sleep. Three days postoperative, he was too distressed with the lack of sleep and the hypnagogic hallucination that he 
started to bang his head to the wall. He denied suicidal intention and yet his judgement was impaired by exhibiting self-injurious behaviour. There was no fluctuation in his conscious level or disorganised thought. He denied manic or depressive symptoms. The pain was well-controlled with Oral Etoricoxib 120mg omne in die and Oral Paracetamol $1 \mathrm{~g}$ quarter in die. He never had any sleep problem prior to this incident.

He has had history of taking 'ecstasy' and alcohol consumption two to three times per month. The last intake was 3 months prior to the operation. He was not on any medication for the last month. Urine for substances was done to exclude illicit substance use. Only morphine was positive in which given on the day of operation.

He was given a symptomatic treatment to help him sleep which were Oral Quetiapine 50mg omne nocte and Oral Lorazepam $1 \mathrm{mg}$ pro re nata. His sleep improved gradually. However, in the beginning, the Quetiapine was not sufficient and the patient had to take Lorazepam for the first three days to get to sleep. But subsequently, he only took Quetiapine and was able to stop after a week's period. The hypnagogic hallucination resolved completely after day three of medication. Medication was discontinued after one week. He still had difficulty falling asleep but successfully overcame the problem with sleep hygiene practice. After three weeks period, his sleep pattern was back to normal.

\section{DISCUSSION}

Sleep is an unarguably important circadian rhythmicity in every human's life. ${ }^{2}$ Normal sleep comprises of rapid eye movement (REM) sleep and non-rapid eye movement (N-REM) sleep stages. It is very important to have adequate time for each stages of sleep because the secretion of hormones such as growth hormone, prolactin, luteinizing hormone, and melatonin are directly associated with those stages. ${ }^{3}$ Surgeries done under general anaesthesia disturb the normal stages of sleep such as vanishing REM sleep, shorter time of total sleep, reduce amount of slow wave sleep, and prolong $\mathrm{N}$ REM sleep. ${ }^{1}$

Every patient is at risk of sleep disorder notwithstanding their premorbid condition prior to general anaesthesia. A study comparing sleep between patients with and without treated obstructive sleep apnoea underwent anaesthesia for multiple surgical disciplines found that after seven days post-surgery, patients without preexisting obstructive sleep apnoea had less sleep time and poorer sleep efficiency. This could be attributed to the treatment they were already on for the obstructive sleep apnoea indirectly treating the sleep disorder post operatively. Impaired post anaesthesia sleep were related to difficult surgery, pain, psychological, and environmental factors. ${ }^{4}$

As for the patient discussed above, the pain factor has already been eliminated, and he was not having any psychosocial issues at the moment. He was discharged home with good social support. The only explanation remained was the procedure done under general anaesthesia.

Propofol, fentanyl, and morphine are drugs known to induce sleep but at the same time can cause insomnia through their withdrawal or paradoxical effects. ${ }^{4}$ Withdrawal is unlikely in this patient as the medication given was only a stat dose and within therapeutic dosage. The patient was also already free from other recreational drugs for more than two months.

Paradoxical reaction is not alien in the field of medicine. Various reports on multiple classes of medication ranging from antibiotics to antipsychotics had been reported to cause paradoxical reaction whereby the opposite of the expected treatment outcome happens to the patient. ${ }^{4}$ But the most important thing is for the attending physician to be vigilant for this reaction aseach patient may respond differently towards the same treatment. Failure of early detection may result in anxiety symptoms, depressed mood, substance use for self-treating, and worst of all suicide to end the suffering arises from insomnia. ${ }^{6,7}$

Sleep deprivation resulted in global impairment could impair one's quality of life including their social functioning, mental health, physical health, and low satisfaction in general health perception. ${ }^{8}$ Its impact can range from mild impairment to devastating effect like in this case. Hypnagogic hallucination is a true hallucination that occurs during transition between wakefulness and sleep. It is usually caused by sleep disorder rather than causing sleep disorder and commonly seen in 
narcolepsy. It usually does not cause significant distress to those experiencing it. ${ }^{9}$ But due to the unnatural cause of sleep deprivation and hypnagogic hallucination in this case, he found the experience very distressing which resulted in selfinjurious behaviour. This behaviour was attributed to poor judgement resulting from impaired cognition secondary to sleep deprivation. ${ }^{2}$ It was decided that the appropriate method to restore his cognition is to return back the good quality and quantity of sleep.

Treatment consists of non-pharmacological and pharmacological approaches. As in this patient, the severity and devastating experience due to the insomnia warranted both to be initiated at the point he was attended. Medications initiated were Oral Quetiapine $50 \mathrm{mg}$ omne nocte and Oral Lorazepam $1 \mathrm{mg}$ pro re nata. Antipsychotic was chosen due to the distressing hypnagogic hallucination experienced by the patient in addition to the desired sedative effect of Quetiapine. Quetiapine causes sedation mainly by its antihistaminergic properties. Low dose Quetiapine had been used off-label for chronic insomnia without other psychiatric diagnosis but the side effects seem to outweigh its benefit in long-term use. $^{10}$

The aim was a short course of treatment as sleep disorder post anaesthesia is a temporary condition. After a week's period when the sleep disorder has improved, the patient was able to successfully stop the oral medications and practice solely on sleep hygiene method for a good night sleep. He regained back his normal sleep pattern after three weeks.

It is very important for the primary team to recognise the sleep difficulty faced by these patients so that early intervention can be initiated. These patients might still be in the ward, intensive care unit, or already discharged home. Patients might not voluntarily inform the attending physician regarding the sleep problem if they do not know that it is directly associated with the procedure performed prior to it. The primary team especially the anesthetist and the surgeon are recommended to voluntarily ask the patient regarding sleep problem and its severity. Referral to a psychiatrist is a step forward in successful and effective multidisciplinary approach of complicated sleep disorder post anaesthesia.

\section{CONCLUSION}

Sleep disorder is a common complication following surgery under general anaesthesia. It remains under-treated due to its subjective experience. Nonetheless, the effect to the patient can be devastating although it could be easily managed with appropriate pharmacological and nonpharmacological approaches. It is important to explore insomnia post anaesthesia, and it should be treated with a multidisciplinary approach involving respective primary team and psychiatry.

\section{REFERENCES}

1. Rosenberg-Adamsen $\mathrm{S}$, Kehlet $\mathrm{H}$, Dodds $\mathrm{C}$ et al. Postoperative Sleep Disturbances: Mechanisms and Clinical Implications. Br J Anaesth 1996; 76:552-9.

2. Maire M, Reichert CF, Schmidt C. Sleep-wake Rhythms and Cognition. Journal of Cognitive and Behavioral Psychotherapies 2013; 13:133-170.

3. Hauser SL, Josephson SA, eds. Harrison's Neurology in Clinical Medicine. $3^{\text {rd }}$ ed. China; McGraw Hill: 2013.

4. Chung $F$, Liao $P$, Yegneswaran B, Shapiro $C M$, Kang W. Postoperative Changes in Sleepdisordered Breathing and Sleep Architecture in Patients with Obstructive Sleep Apnea.

Anesthesiology 2014; 120:287-98.

5. Smith SW, Hauben M, Aronson JK. Paradoxical and Bidirectional Drug Effects. Drug Saf 2012; 35:173-89.

6. Colten HR, Altevogt BM. Sleep Disorders and Sleep Deprivation: An Unmet Public Health Problem. Washington; National Academies Press: 2006.

7. Liu X. Sleep and Adolescent Suicidal Behavior. Sleep 2004; 27:1351-8.

8. Roth D. Insomnia: Definition, Prevalence, Etiology, and Consequences. J Clin Sleep Med 2007; 3:7-10.

9. Casey P, Kelly B. Fish's Clinical Psychopathology: Signs and Symptoms in Psychiatry. $3^{\text {rd }}$ ed. Glasgow; Bell \& Bain Ltd: 2007.

10. Coe HV, Hong IS. Safety of Low Doses of Quetiapine When Used for Insomnia. Ann Pharmacother 2012; 46:718-22. 
